\title{
Synthesis, X-Ray Structure Determination and Related Physical Properties of Thiazolidinone Derivative by DFT Quantum Chemical Method
}

\author{
Youcef Megrouss, ${ }^{1, \star}$ Fayssal Triki Baara, ${ }^{2}$ Nourdine Boukabcha, ${ }^{1}$ \\ Abdelkader Chouaih, ${ }^{1}$ Antonis Hatzidimitriou, ${ }^{3}$ Ayada Djafri, ${ }^{2}$ Fodil Hamzaoui ${ }^{4}$ \\ ${ }^{1}$ LTPS Laboratory, University Abdelhamid Ibn Badis - Mostaganem, 27000 Mostaganem, Algeria \\ ${ }^{2}$ Laboratory of Applied Organic Synthesis (LSOA), Department of Chemistry, Faculty of Sciences, University \\ of Oran-1 Ahmed Ben Bella, 31000 Oran, Algeria \\ ${ }^{3}$ Department of General and Inorganic Chemistry, Faculty of Chemistry, Aristotle University of Thessaloniki, \\ GR-54124 Thessaloniki, Greece \\ ${ }^{4}$ LPFM Académie de Montpellier, France \\ *Corresponding author: E-mail: youmeg@hotmail.fr
}

Received: 02-20-2019

\begin{abstract}
In this paper we report the synthesis and characterization of the (Z)-3-N-(ethyl)-2- $N^{3}-((3-$ methoxyphenyl)imino)thiazolidine-4-one by means of FT-IR, ${ }^{1} \mathrm{H}$ and ${ }^{13} \mathrm{C}$ NMR and by single crystal X-ray diffraction. The experimental determination of the crystal structure of the compound has been achieved using X-ray diffraction data. The important characteristic of the structure is the existence of a dihedral angle formed by the benzene and thiazolidinone rings being equal to $86.0^{\circ}$ indicating an absence of $\pi-\pi$ stacking as well as that the structure is non planar. In the crystal, the molecules are linked by $\mathrm{C}-\mathrm{H} \cdots \mathrm{O}$ and $\mathrm{C}-\mathrm{H} \cdots \mathrm{N}$ hydrogen bonds, these bonds being responsible for the three-dimensional molecular structure packing. In order to compare the experimental results with those of the theoretical calculation, quantum chemical DFT calculations were carried out using B3LYP/6-311G(d,p) basis set. In this context, the molecular electrostatic potential around the molecule and HOMO-LUMO energy levels were also computed. The dipole moment orientations were determined in order to understand the nature of inter- and intramolecular charge transfer. Finally, the stability of the title compound was confirmed throughout the calculation of the chemical reactivity descriptors.
\end{abstract}

Keywords: X-ray diffraction; single crystal; DFT calculations; spectroscopy; FT-IR; ${ }^{1} \mathrm{H}$ and ${ }^{13} \mathrm{C}$ NMR.

\section{Introduction}

Heterocyclic compounds are the major family of organic compounds; in medicinal chemistry thiazole derivatives are of great importance for their chemical and pharmacological properties, consequently thiazole derivatives have an extended range of pharmacological applications. Over the years, interesting biological activities were combined with thiazole derivatives. ${ }^{1-2}$ These materials are extremely necessary with wide range of synthetic, pharmaceutical and industrial applications and are famous for their biological activities. ${ }^{3,4}$ Recently, in drug development the application of thiazoles was required for the treatment of allergies, ${ }^{5}$ hypertension, ${ }^{6}$ inflammation, ${ }^{7}$ schizophrenia, ${ }^{8}$ bacterial ${ }^{9}$ and HIV infections, ${ }^{10}$ as hypnotics ${ }^{11}$ and more recently for pain treatment, ${ }^{12}$ as fibrinogen receptor antagonists with antithrombotic activity. ${ }^{13}$ In addition, thiazolidinones and thiazoles present a very powerful activity as anti mycobacterium in tuberculosis. ${ }^{14}$ In this context, we have tried to realize the synthesis of the title compound, to characterize and perform its structural analysis as well as the theoretical density functional theory (DFT) calculations. In this work we present the synthesis, single crystal structure, IR and NMR spectroscopic characterizations as well as DFT calculations of this new thiazole derivative compound, namely the (Z)-3-N-(ethyl)-2-N"-((3-methoxyphenyl)imi- 
no)thiazolidine-4-one. Furthermore, theoretical vibrational frequencies and ${ }^{1} \mathrm{H}$ and ${ }^{13} \mathrm{C}$ NMR chemical shifts were calculated and compared to the experimental values. Additional parameters as molecular orbitals and chemical reactivity descriptors are evaluated in order to confirm the stability of the title compound. Finally, the molecular electrostatic potential was computed to determine electrophilic and nucleophilic regions of the title molecule.

\section{Experimental}

\section{1. Synthesis and Crystallization}

An equimolar solution of $N$-ethyl-3- $N$-(3-methoxyphenyl)thiourea and ethyl bromoacetate in absolute ethanol in the presence of sodium acetate was refluxed for $6 \mathrm{~h}$. The solvent was removed by vacuum distillation and the residue was isolated, washed with cold water, filtered, dried and crystallized from ethanol to yield (Z)-3-N-(ethyl)-2$N^{3}$-((3-methoxyphenyl)imino)thiazolidin-4-one as presented in the scheme 1 .

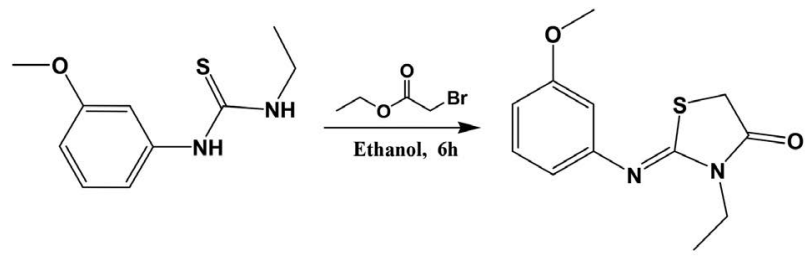

Scheme 1. Reaction sequence for the title compound synthesis.

\section{2. Spectral Data Measurements}

Infrared (IR) spectrum of the molecule (Z)-3-N(ethyl)-2-N'-((3-methoxyphenyl)imino)thiazolidine-4-one was recorded in the range $500-4000 \mathrm{~cm}^{-1}$ on a Nicolet FT-IR 6700 spectrometer using sample prepared as KBr pellets. ${ }^{1} \mathrm{H}$ NMR $(500 \mathrm{MHz})$ and ${ }^{13} \mathrm{C}$ NMR $(125 \mathrm{MHz})$ spectra of the molecule using $\mathrm{CDCl}_{3}$ as the solvent were recorded on Bruker AC250 spectrometer at $298 \mathrm{~K}$.

\section{3. X-Ray Data Collection and Processing}

A transparent-yellowish parallelepiped crystal was selected and separated from the mother liquor, immediately cooled to $130 \mathrm{~K}$ and mounted on a Bruker Kappa APEX 2 diffractometer, equipped with a Triumph monochromator using MoKa radiation. The crystal presented no decay during the data collection. The frames collected (running $\phi$ and $\omega$ scans) were integrated with the Bruker SAINT Software package, ${ }^{15}$ using a narrow-frame algorithm. Data were corrected using the SADABS program. ${ }^{16}$ The structure was solved by the SUPERFLIP package. ${ }^{17}$ Crystals program package version $14.40^{18}$ has been used for the refinement and all the rest subsequent calculations through full-matrix least-squares on $F^{2}$. All non-hydrogen atoms have been refined anisotropically. Hydrogen atoms were found at their expected positions and refined using proper riding constraints to the pivot atoms. Molecular illustrations were made through the MoPro-viewer crystallographic program. ${ }^{19}$ Crystallographic and experimental details are summarized in Table 1.

Table 1. Crystallographic and experimental details.

\begin{tabular}{|c|c|}
\hline \multicolumn{2}{|l|}{ Crystal data } \\
\hline Chemical formula & $\mathrm{C}_{12} \mathrm{H}_{14} \mathrm{~N}_{2} \mathrm{O}_{2} \mathrm{~S}$ \\
\hline$M_{\mathrm{r}}$ & 250.32 \\
\hline Crystal system & Monoclinic \\
\hline Space group & $\mathrm{C} 2 / \mathrm{c}$ \\
\hline$a(\AA)$ & $23.7067(19)$ \\
\hline$b(\AA)$ & $6.8884(6)$ \\
\hline$c(\AA)$ & $15.7244(12)$ \\
\hline$\beta\left({ }^{\circ}\right)$ & $111.6504(17)$ \\
\hline$V\left(\AA^{3}\right)$ & $2386.66(18)$ \\
\hline$Z$ & 8 \\
\hline$\mu\left(\mathrm{mm}^{-1}\right)$ & 0.26 \\
\hline Crystal size (mm) & $0.31 \times 0.28 \times 0.19$ \\
\hline Diffractometer & Bruker Kappa Apex2 \\
\hline \multirow[t]{2}{*}{ Absorption correction } & Numerical \\
\hline & $\begin{array}{l}\text { Analytical Absorption } \\
\text { (De Meulenaer \& Tompa, 1965) }\end{array}$ \\
\hline$T_{\min }, T_{\max }$ & $0.93,0.95$ \\
\hline Radiation type, $\lambda(\AA)$ & Mo Ka, 0.71073 \\
\hline Temperature $(\mathrm{K})$ & 130 \\
\hline Measured & 17268 \\
\hline \multicolumn{2}{|l|}{ Independent } \\
\hline Observed $[I>2.0 \sigma(I)]$ & 3006 \\
\hline \multicolumn{2}{|l|}{2843} \\
\hline$R_{\text {int }}$ & 0.015 \\
\hline$R\left[F^{2}>2 \sigma\left(F^{2}\right)\right]$ & 0.029 \\
\hline$w R\left(F^{2}\right)$ & 0.066 \\
\hline$S$ & 1.00 \\
\hline No. of reflections & 2843 \\
\hline No. of parameters & 154 \\
\hline$\Delta \rho_{\max }, \Delta \rho_{\min }\left(\mathrm{e} \AA^{-3}\right)$ & $0.33,0.21$ \\
\hline
\end{tabular}

\section{DFT Calculations}

In this theoretical study, the hybrid functional B3LYP with the 6-311G $(\mathrm{d}, \mathrm{p})^{20}$ basis set were used in all calculations by using the Gaussian 09 program, ${ }^{21}$ the $\mathrm{X}$-ray structure was used as starting geometry to optimize a molecular structure of the investigation compound. Vibrational frequencies were calculated and the Gauss-view molecular visualization program ${ }^{22}$ and VEDA software ${ }^{23}$ were used for the assignment. Additionally, ${ }^{1} \mathrm{H}$ and ${ }^{13} \mathrm{C}$ NMR chemical shifts were calculated using the same level of theory. The theoretical calculation also allowed us to compute the energy values of the highest occupied molecular orbital (HOMO) and lowest unoccupied molecular orbital (LUMO). Furthermore, the molecular electrostatic 
potential was calculated with B3LYP/6-311G(d, p) to highlight the electrophilic and nucleophulic attack sites.

\section{Results and Discussion}

\section{1. Structure Description}

The molecular geometry of the title compound is defined by the presence of two fragments, methoxyphenyl and thiazolidine rings forming a dihedral angle of $86^{\circ}$. Detailed results containing atomic positions and thermal parameters are given in the CIF file. Selected bond lengths, bond and torsion angles for all atoms by X-ray diffraction and theoretical calculations are listed in Tables 2, 3 and 4, respectively. An ORTEPIII diagram ${ }^{24}$ of the title compound showing the $\mathrm{X}$-ray structure with thermal ellipsoids of the different atoms and the theoretical structure are given in Figure 1. In our study we employed full geometry optimization for the molecule without symmetry constraint. The results of our calculations showed that $\mathrm{S} 1-\mathrm{C} 5, \mathrm{~S} 1-\mathrm{C} 1, \mathrm{C} 1-\mathrm{N} 1, \mathrm{~N} 1-\mathrm{C} 2$, $\mathrm{N} 1-\mathrm{C} 4, \mathrm{C} 6-\mathrm{N} 2$ and $\mathrm{O} 2-\mathrm{C} 8$ bonds exhibit single bond characteristics, while C4-O2 (1.23 $\mathrm{A})$ and N2-C1 (1.26 $)$ bonds show typical double bond characteristics. ${ }^{25}$ The amine $\mathrm{N} 2$ atom exhibits a geometry that is typical for an $\mathrm{sp}^{2}$ rather than an $\mathrm{sp}^{3}$ atom hybridisation. In addition, the difference in the thiazole ring bonds between theoretical calculation and experimental values does not exceed $0.13 \AA$. Bond angles C6-N2-C1, C2-N1-C4 and $\mathrm{C} 1-\mathrm{N} 1-\mathrm{C} 4$ are also near $120^{\circ}\left(119.56^{\circ}, 121.08^{\circ}\right.$ and $116.72^{\circ}$, respectively $){ }^{26}$

a)

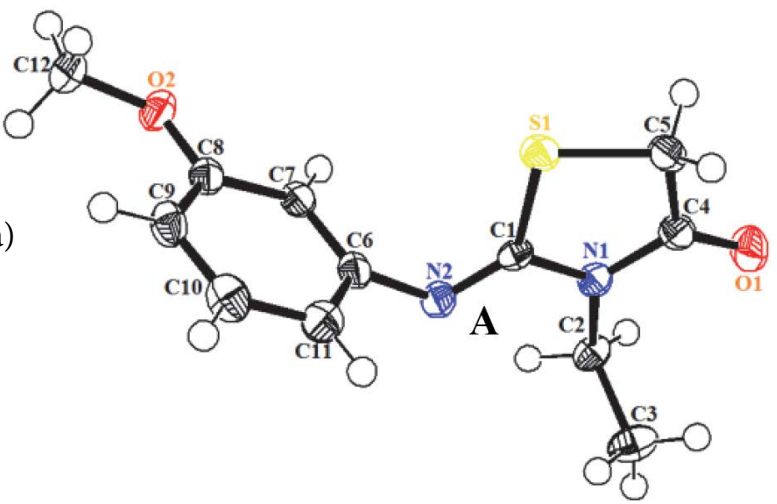

b)

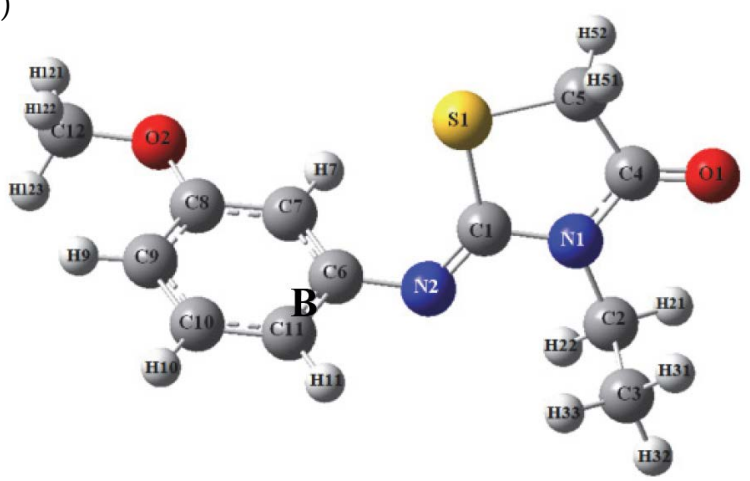

Figure 1. Experimental (a) and theoretical (b) structure of (Z)-3-N(ethyl)-2- $N$-((3-methoxyphenyl)imino)thiazolidine-4-one.
The bond angles centered on $\mathrm{C} 1$ are all between $109^{\circ}$ and $129^{\circ}$. The $\mathrm{C} 1$ atom is of $\mathrm{sp}^{2}$ hybridization type because the total adds up to $360^{\circ}$ at $\mathrm{B} 3 \mathrm{LYP} / 6-311 \mathrm{G}(\mathrm{d}, \mathrm{p})$ level. The corresponding theoretical values of this angle is $64^{\circ}(\mathrm{C} 7-\mathrm{C} 6-\mathrm{N} 2-$ $\mathrm{C} 1)$. The $\mathrm{C} 8-\mathrm{O} 2-\mathrm{C} 12$ angle is $116.98^{\circ}$, and the $\mathrm{C} 2-\mathrm{N} 1-\mathrm{C} 4-$ O1 fragment is approximately planar (Table 4).

Generally, the observed difference between experimental and calculated geometrical parameters does not seem very large.

Table 2. Experimental and optimized bond lengths ( $\mathrm{A})$.

\begin{tabular}{lcc}
\hline Bond lengths & X-ray & B3LYP/6-311G(d,p) \\
\hline S1-C5 & $1.811(1)$ & 1.887 \\
S1-C1 & $1.767(1)$ & 1.893 \\
O1-C4 & $1.216(1)$ & 1.233 \\
N1-C2 & $1.469(1)$ & 1.485 \\
N1-C1 & $1.399(1)$ & 1.392 \\
N1-C4 & $1.371(1)$ & 1.388 \\
N2-C6 & $1.425(1)$ & 1.407 \\
N2-C1 & $1.263(1)$ & 1.264 \\
O2-C8 & $1.367(1)$ & 1.386 \\
O2-C12 & $1.431(1)$ & 1.459 \\
C6-C7 & $1.389(1)$ & 1.398 \\
C6-C11 & $1.402(1)$ & 1.408 \\
C7-C8 & $1.398(1)$ & 1.401 \\
C5-C4 & $1.509(1)$ & 1.527 \\
C2-C3 & $1.518(1)$ & 1.537 \\
C10-C9 & $1.393(1)$ & 1.399 \\
C10-C11 & $1.386(1)$ & 1.387 \\
C8-C9 & $1.395(1)$ & 1.397 \\
\hline
\end{tabular}

Table 3. Experimental and optimized bond angles $\left({ }^{\circ}\right)$.

\begin{tabular}{lrc}
\hline Bond angles & X-ray & B3LYP/6-311G(d,p) \\
\hline C5-S1-C1 & 92.24 & 90.13 \\
C2-N1-C1 & 122.15 & 120.13 \\
C2-N1-C4 & 121.08 & 119.58 \\
C1-N1-C4 & 116.72 & 29.83 \\
C6-N2-C1 & 119.56 & 129.18 \\
C8-O2-C12 & 116.98 & 117.97 \\
N2-C6-C7 & 119.43 & 124.07 \\
N2-C6-C11 & 119.94 & 116.82 \\
C7-C6-C11 & 120.34 & 119.08 \\
C6-C7-C8 & 119.87 & 120.72 \\
S1-C5-C4 & 107.51 & 108.27 \\
N1-C2-C3 & 112.36 & 111.42 \\
S1-C1-N1 & 110.92 & 109.07 \\
S1-C1-N2 & 127.32 & 129.12 \\
N1-C1-N2 & 121.76 & 121.81 \\
O1-C4-N1 & 123.71 & 123.73 \\
O1-C4-C5 & 123.91 & 124.08 \\
N1-C4-C5 & 112.37 & 112.18 \\
C9-C10-C11 & 121.52 & 120.98 \\
O2-C8-C7 & 115.23 & 115.22 \\
O2-C8-C9 & 124.48 & 124.87 \\
C7-C8-C9 & 120.27 & 119.91 \\
C10-C9-C8 & 119.01 & 119.34 \\
C6-C11-C10 & 118.98 & 119.96 \\
\hline
\end{tabular}


Table 4. Experimental and optimized torsion angles $\left({ }^{\circ}\right)$.

\begin{tabular}{|c|c|c|}
\hline Torsion angles & X-ray & B3LYP/6-311G (d,p) \\
\hline $\mathrm{C} 1-\mathrm{S} 1-\mathrm{C} 5-\mathrm{C} 4$ & 3.6 & 2.4 \\
\hline $\mathrm{C} 5-\mathrm{S} 1-\mathrm{C} 1-\mathrm{N} 1$ & -4.7 & -2.4 \\
\hline $\mathrm{C} 5-\mathrm{S} 1-\mathrm{C} 1-\mathrm{N} 2$ & 174.5 & 176.7 \\
\hline $\mathrm{C} 1-\mathrm{N} 1-\mathrm{C} 2-\mathrm{C} 3$ & -99.6 & -86.2 \\
\hline $\mathrm{C} 4-\mathrm{N} 1-\mathrm{C} 2-\mathrm{C} 3$ & 77.9 & 92.2 \\
\hline $\mathrm{C} 2-\mathrm{N} 1-\mathrm{C} 1-\mathrm{S} 1$ & -177.7 & -179.7 \\
\hline $\mathrm{C} 2-\mathrm{N} 1-\mathrm{C} 1-\mathrm{N} 2$ & 3.1 & 1.1 \\
\hline $\mathrm{C} 4-\mathrm{N} 1-\mathrm{C} 1-\mathrm{S} 1$ & 4.7 & 1.8 \\
\hline $\mathrm{C} 4-\mathrm{N} 1-\mathrm{C} 1-\mathrm{N} 2$ & -174.6 & -177.3 \\
\hline $\mathrm{C} 2-\mathrm{N} 1-\mathrm{C} 4-\mathrm{O} 1$ & -0.2 & 1.7 \\
\hline $\mathrm{C} 2-\mathrm{N} 1-\mathrm{C} 4-\mathrm{C} 5$ & -179.5 & -178.4 \\
\hline $\mathrm{C} 1-\mathrm{N} 1-\mathrm{C} 4-\mathrm{O} 1$ & 177.4 & -179.9 \\
\hline $\mathrm{C} 1-\mathrm{N} 1-\mathrm{C} 4-\mathrm{C} 5$ & -1.9 & 0.1 \\
\hline C1-N2-C6-C7 & 86.0 & 64.5 \\
\hline C1-N2-C6-C11 & -100.2 & -119.3 \\
\hline C6-N2-C1-S1 & 1.6 & 4.4 \\
\hline C6-N2-C1-N1 & -179.2 & -176.7 \\
\hline $\mathrm{C} 12-\mathrm{O} 2-\mathrm{C} 8-\mathrm{C} 7$ & -170.8 & 179.8 \\
\hline $\mathrm{C} 12-\mathrm{O} 2-\mathrm{C} 8-\mathrm{C} 9$ & 10.9 & -0.1 \\
\hline N2-C6-C7-C8 & 172.3 & 178.5 \\
\hline C11-C6-C7-C8 & -1.5 & 0.6 \\
\hline N2-C6-C11-C10 & -173.1 & -179.4 \\
\hline C7-C6-C11-C10 & 0.6 & -1.3 \\
\hline $\mathrm{C} 6-\mathrm{C} 7-\mathrm{C} 8-\mathrm{O} 2$ & -177.2 & -179.5 \\
\hline C6-C7-C8-C9 & 1.3 & 0.4 \\
\hline $\mathrm{S} 1-\mathrm{C} 5-\mathrm{C} 4-\mathrm{O} 1$ & 178.9 & 178.06 \\
\hline $\mathrm{S} 1-\mathrm{C} 5-\mathrm{C} 4-\mathrm{N} 1$ & -1.8 & -1.9 \\
\hline C11-C10-C9-C8 & -0.8 & -0.1 \\
\hline C9-C10-C11-C6 & 0.5 & 1.1 \\
\hline $\mathrm{O} 2-\mathrm{C} 8-\mathrm{C} 9-\mathrm{C} 10$ & 178.2 & 179.2 \\
\hline C7-C8-C9-C10 & -0.1 & -0.7 \\
\hline
\end{tabular}

\section{2. Hydrogen Bonding}

Hydrogen bonds join chains of molecules to stabilize the crystal structure of the title compound. Along the $b$ axis in the unit cell, the translation of equivalent molecules allows the linking of the almost linear hydrogen bonding. $\mathrm{C}-\mathrm{H} \cdots \mathrm{O}$ and $\mathrm{C}-\mathrm{H} \cdots \mathrm{N}$ intra- and intermolecular interactions are present in the crystal structure. The molecular conformation is in part influenced by the formation of two weak intramolecular $\mathrm{C} 2-\mathrm{H} 22 \cdots \mathrm{O} 1$ and $\mathrm{C} 2-\mathrm{H} 21 \cdots \mathrm{N} 1$ hydrogen bonds that enclose S(5) rings (Figure 2 and Table 5). a)

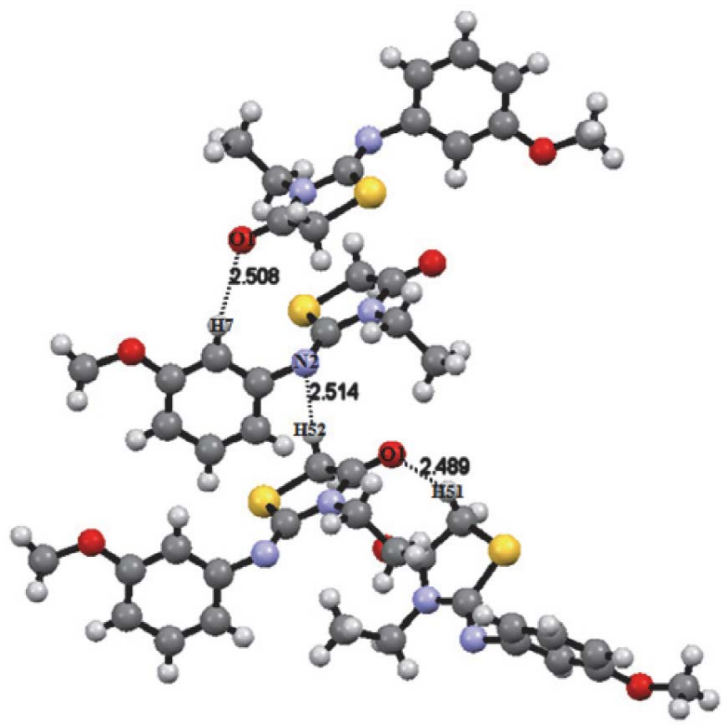

b)

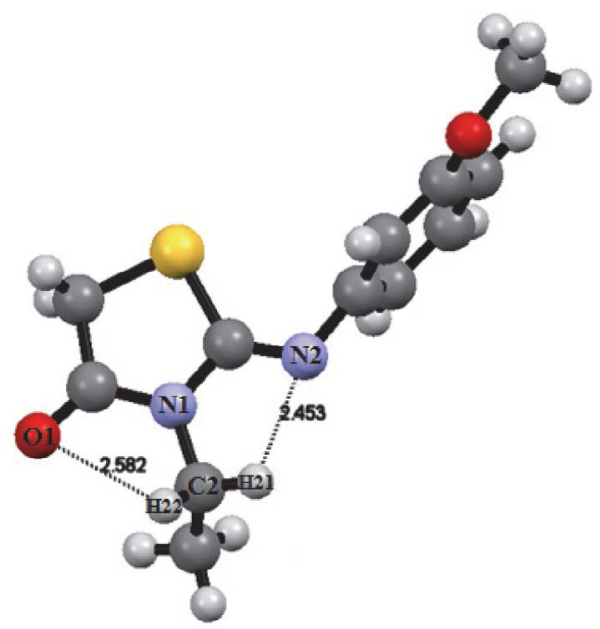

Figure 2. Hydrogen bonding view in the crystal showing: (A) intermolecular interactions, (B) $\mathrm{C} 2-\mathrm{H} 21 \cdots \mathrm{N} 2$ and $\mathrm{C} 2-\mathrm{H} 22 \cdots \mathrm{O} 1$ intramolecular interactions.

These interactions are responsible for the stability of the molecular packing, as the $\mathrm{C} 2, \mathrm{C} 3, \mathrm{C} 5, \mathrm{C} 12$ and $\mathrm{C} 7$ carbon atoms act as donor groups and both oxygen and nitrogen atoms play the acceptor role. Hydrogen bond interactions are presented in Table 5. Figure 2 shows all interaction types in the crystal. The molecular stacking that has been provided by the different existing hydrogen bonds in the

Table 5. Geometry of the $\mathrm{C}-\mathrm{H} \cdots \mathrm{O}$ and $\mathrm{C}-\mathrm{H} \cdots \mathrm{N}$ hydrogen bonds in (Z)-3-N-(ethyl)-2-N'-((3-methoxyphenyl)imino)thiazolidine-4-one crystal by $\mathrm{X}$-ray diffraction.

\begin{tabular}{|c|c|c|c|c|c|}
\hline $\mathrm{D}-\mathrm{H} \cdots \mathrm{A}$ & D-H (̊̊) & $\mathrm{D}-\mathrm{A}(\AA)$ & $\mathrm{H} \cdots \mathrm{A}(\AA)$ & $\mathrm{D}-\mathrm{H} \cdots \mathrm{A}\left({ }^{\circ}\right)$ & Bond type \\
\hline $\mathrm{C} 2-\mathrm{H} 21 \cdots \mathrm{N} 2$ & 0.981 & 2.852 & 2.453 & 103.88 & Intramolecular \\
\hline $\mathrm{C} 2-\mathrm{H} 22 \cdots \mathrm{O} 1$ & 0.962 & 2.817 & 2.582 & 93.97 & Intramolecular \\
\hline $\mathrm{C} 5-\mathrm{H} 51 \cdots \mathrm{O} 1^{(i)}$ & 0.987 & 3.380 & 2.489 & 149.92 & Intermolecular \\
\hline $\mathrm{C} 7-\mathrm{H} 7 \ldots \mathrm{O} 1^{(i i)}$ & 0.947 & 3.432 & 2.508 & 164.98 & Intermolecular \\
\hline $\mathrm{C} 5-\mathrm{H} 52 \cdots \mathrm{N} 2^{(i i i)}$ & 0.955 & 3.370 & 2.514 & 149.26 & Intermolecular \\
\hline
\end{tabular}

Symmetry codes: (i) $-x, y,-z+1 / 2$; (ii) $-x,-y,-z$; (iii) $x,-y, z+1 / 2$ 


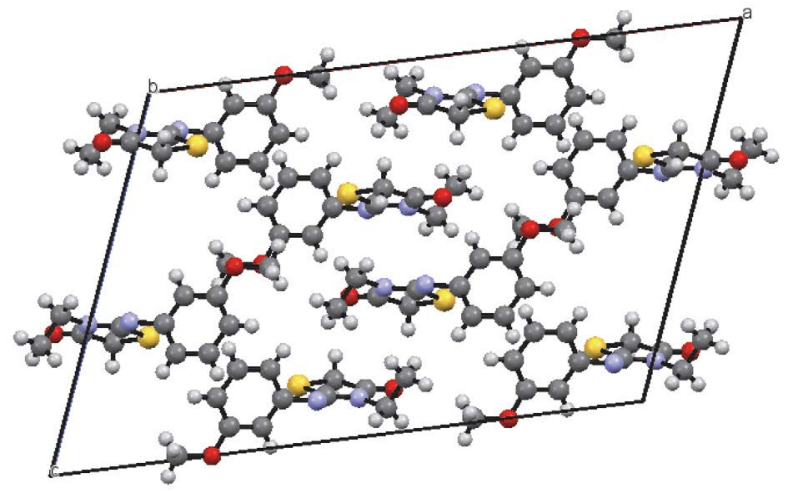

Figure 3. View of the crystal packing along the $b$ axis of (Z)-3-N(ethyl)-2- $N^{3}$-((3-methoxyphenyl)imino)thiazolidine-4-one molecule.

crystal is shown in Figure 3. This figure shows the existence of eight molecules in the unit cell which is in good agreement with the multiplicity of the space group $\mathrm{C} 2 / \mathrm{c}$.

\section{3. Vibrational Frequencies Assignments}

Using analytic second derivatives to validate the convergence to minima on the potential energy surface the fundamental frequencies of the studied molecule have been calculated to understand the nature of these modes of vibration IR absorption spectroscopy based on DFT calculation. A theoretical analysis has been realized using B3LY$\mathrm{P} / 6-311 \mathrm{G}(\mathrm{d}, \mathrm{p})$ level of theory in gas phase. The probable assignments were performed by means of VEDA 4 program. ${ }^{23}$ The vibrational frequencies obtained from B3LYP functional calculations have been scaled by a factor of $0.967 .{ }^{27}$ Table 6 shows the calculated (unscaled and scaled) and experimental frequencies of the title compound. Simulated and experimental IR spectra of (Z)-3-N-(ethyl)-2- $N$ ((3-methoxyphenyl)imino)thiazolidine-4-one are shown in Figure 4. As can be seen in Figure 4, the experimental fundamentals are in better agreement with the scaled fundamentals.

\section{3. 1. Carbon-Hydrogen Vibrations}

In the aromatic compounds, multiple weak bands are exhibited in the region of $3100-3000 \mathrm{~cm}^{-1}$. 28 They appear in this range of like multiple weak bands due to the stretching vibrations of $\mathrm{C}-\mathrm{H} .{ }^{29}$ In the present work, the carbon-hydrogen of aromatic ring $(\mathrm{C}-\mathrm{H})$ stretching $(\mathrm{vCH}$ modes) were found in a range of 3000 and 3100 $\mathrm{cm}^{-1}$ using the B3LYP/6-311G(d,p) calculations. These modes involve exact contribution of $>91 \%$ suggesting that they are pure stretching modes. The $\mathrm{C}-\mathrm{H}$ stretching of aromatic ring showed symmetric modes at 3001,3070 and $3080 \mathrm{~cm}^{-1}$ while asymmetric stretching mode was observed at $2989 \mathrm{~cm}^{-1}$.

\section{3. 2. $\mathrm{C}=\mathrm{O}$ and $\mathrm{C}=\mathrm{N}$ Vibrations}

Usually, the $\mathrm{C}=\mathrm{O}$ stretching vibration mode can be easily observed as a strong band in the region 1850-1550 $\mathrm{cm}^{-1}{ }^{30}$ The $\pi-\pi$ bonding between carbon and oxygen is responsible for forming a double bond between the carbon and oxygen atoms. The electronic distribution in this link is not equal because these atoms have different electronegativities. The lone pair of electrons on oxygen is responsible for the polar nature of the carbonyl group. In the present study, the single $\mathrm{C}=\mathrm{O}$ stretching vibration mode was observed as a high-intense peak in FT-IR at $1716 \mathrm{~cm}^{-1}$ while the calculated value shows at $1726 \mathrm{~cm}^{-1}$ with a PED (potential energy distribution) of $79 \%$. The stretching frequency of the double bond $\mathrm{C}=\mathrm{N}$ is perfectly observed at the high absorption $1640 \mathrm{~cm}^{-1}$. DFT/B3LYP functional with $6-311 \mathrm{G}(\mathrm{d}, \mathrm{p})$ basis set gives exactly the same value of $1640 \mathrm{~cm}^{-1}$ for the $\mathrm{C}=\mathrm{N}$ vibration.

\section{3. 3. Thiazolidine Ring Vibrations}

The C-S stretching vibration presents average bands in the region $1020-1010 \mathrm{~cm}^{-1} \cdot{ }^{31}$ In this paper C-S vibrations were observed at 704,647 and $526 \mathrm{~cm}^{-1}$. The examination of the theoretical results gives us the following values with their PED contribution: 720 (21\%), $642(10 \%)$, $522(12 \%)$ and $482(23 \%)$ respectively, which shows a good agreement between theoretical and experimental ones as shown in the Table 6. C-N vibration is a difficult task to identify since the appearance of several bands is possible in the region, Gunasekaran et al. ${ }^{32}$ have observed C-N stretching band at $1312 \mathrm{~cm}^{-1}, 33$ and $\mathrm{C}-\mathrm{N}$ assigned stretching vibration in the region $1350-1000 \mathrm{~cm}^{-1}$ for amines. In thiazolidine, $\mathrm{C}-\mathrm{N}$ stretching band is found to be present at 1382 and $1307 \mathrm{~cm}^{-1}$. The $\mathrm{C}-\mathrm{N}$ stretching vibrations are expected to occur in the region $1200-1130 \mathrm{~cm}^{-1} \cdot{ }^{34}$ In our present work of the title molecule FTIR bands were observed at $896 \mathrm{~cm}^{-1}$ and $1234 \mathrm{~cm}^{-1}$, the theoretically calculated bands at $940,1405,1357,1301,1257,1131$ are shifted

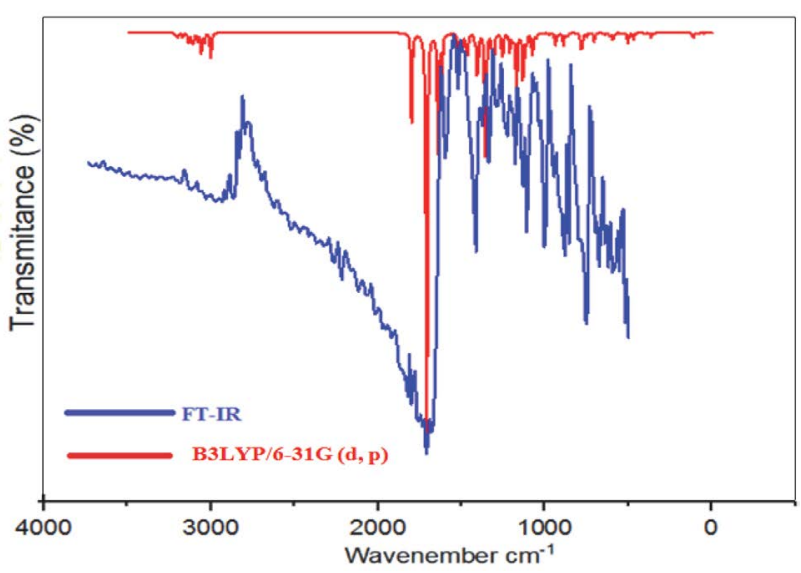

Figure 4. Comparison of FT-IR and calculated IR Spectra for (Z)-3$N$-(ethyl)-2- $N^{\prime}$-((3-methoxyphenyl)imino)thiazolidine-4-one. 
Table 6. Comparison of the calculated harmonic frequencies using B3LYP method 6-311G $(\mathrm{d}, \mathrm{p})$ basis set and experimental (FT-IR) wavenumbers $\left(\mathrm{cm}^{-1}\right)$ for $(Z)-3-N$-(ethyl)-2-N'-((3-methoxyphenyl)imino)thiazolidine-4-one.

\begin{tabular}{|c|c|c|c|c|c|}
\hline \multirow[t]{2}{*}{ No } & \multirow{2}{*}{$\begin{array}{l}\text { Experi- } \\
\text { mental }\end{array}$} & \multicolumn{3}{|c|}{ B3LYP/6-311G(d,p) } & \multirow[t]{2}{*}{ Assignments with PED>10\% } \\
\hline & & Unscaled & Scaled & $\mathrm{I}_{\mathrm{IR}}$ & \\
\hline 87 & & 3209 & 3080.64 & 8.75 & $v \mathrm{CH}(91 \%)$ Ar ring \\
\hline 86 & & 3198 & 3070.08 & 0.77 & $v \mathrm{CH}(92 \%)$ Ar ring \\
\hline 85 & & 3127 & 3001.92 & 9.61 & $v \mathrm{CH}(100 \%)$ Ar ring \\
\hline 83 & & 3139 & 3013.44 & 9.25 & $v \mathrm{CH}(79 \%)+v_{\mathrm{as}} \mathrm{CH}(11 \%)$ \\
\hline 81 & & 3127 & 3001.92 & 0.33 & $v \mathrm{CH}(100 \%)$ \\
\hline 80 & 2971 & 3114 & 2989.44 & 13.61 & $v_{\mathrm{as}} \mathrm{CH}(92 \%)$ \\
\hline 74 & & 3001 & 2880.96 & 61.94 & $v \mathrm{CH}(92 \%)$ \\
\hline 73 & 1716 & 1798 & 1726.08 & 182.70 & $v \mathrm{C}=\mathrm{O}(79 \%)$ \\
\hline 72 & 1640 & 1709 & 1640.64 & 943.72 & $v \mathrm{~N}=\mathrm{C}(73 \%)$ \\
\hline 71 & & 1640 & 1574.4 & 329.64 & $v \mathrm{CC}_{\text {asy }}(63 \%)$ Ar ring $+\delta$ HCC (14\%) Ar ring \\
\hline 70 & & 1617 & 1552.32 & 93.33 & $v$ CC $(51 \%)_{\text {asy }}$ Ar ring $+\delta$ HCC $(11 \%)$ Ar ring $+\delta$ CCC (12\%) Ar ring \\
\hline 69 & 1482 & 1518 & 1457.28 & 72.82 & $\delta \mathrm{HCC}_{\text {asy }}(44 \%)$ Ar ring $+\delta \mathrm{CCC}(19 \%)$ Ar ring \\
\hline 68 & & 1508 & 1447.68 & 33.99 & $\delta \mathrm{HCH}(67 \%)+$ \\
\hline 67 & & 1506 & 1445.76 & 3.59 & $\delta \mathrm{HCH}(71 \%)+\tau \mathrm{HCCN}_{\text {asy }}(22 \%)$ Th ring \\
\hline 66 & & 1498 & 1438.08 & 8.56 & $\delta \mathrm{HCH}(71 \%)+\tau \mathrm{HCCN}_{\text {asy }}(24 \%)$ Th ring \\
\hline 65 & & 1492 & 1432.32 & 7.71 & $\delta \mathrm{HCH}_{\text {asy }}(69 \%)+\tau \mathrm{HCOC}_{\text {asy }}(24 \%)$ Ar ring \\
\hline 64 & & 1482 & 1422.72 & 0.82 & $\delta \mathrm{HCH}(48 \%)$ \\
\hline 62 & & 1465 & 1406.4 & 40.95 & $v \mathrm{CC}_{\text {asy }}(31 \%)$ Ar ring $+\delta \mathrm{HCC}(31 \%)$ Ar ring $+\delta \mathrm{HCC}_{\mathrm{asy}}(16 \%)$ \\
\hline 61 & & 1462 & 1403.52 & 8.14 & $\delta \mathrm{HCH}(88 \%)$ \\
\hline 60 & 1391 & 1420 & 1363.2 & 18.06 & $\delta \mathrm{HCC}(13 \%)+\delta \mathrm{HCH}(15 \%)+\tau \mathrm{HCNC}(46 \%)$ Th ring \\
\hline 59 & & 1405 & 1348.8 & 110.90 & $\begin{array}{l}\nu \mathrm{NC}(13 \%) \text { Th ring }+\delta \mathrm{HCC}(10 \%)+\delta \mathrm{HCH}(39 \%)+\tau \mathrm{HCNC}_{\text {asy }}(10 \%) \\
\text { Th ring }\end{array}$ \\
\hline 58 & & 1401 & 1344.96 & 12.94 & $\delta \mathrm{HCC}(56 \%)+\delta \mathrm{HCH}(14 \%)$ \\
\hline 57 & & 1357 & 1302.72 & 247.21 & $v \mathrm{NC}(46 \%)$ Th ring \\
\hline 56 & 1284 & 1350 & 1296 & 24.41 & $v \mathrm{CC}_{\mathrm{asy}}(59 \%)$ Ar ring $+\delta \mathrm{HCC}(23 \%)$ \\
\hline 55 & & 1312 & 1259.52 & 111.70 & $v \mathrm{CO}_{\mathrm{asy}}(79 \%)$ Ar ring $+\delta \mathrm{HCC}(37 \%)$ Ar ring \\
\hline 54 & 1234 & 1301 & 1248.96 & 13.79 & $\begin{array}{l}v \mathrm{CC}(10 \%) \text { Ar ring }+v \mathrm{NC}_{\mathrm{th}}(12 \%) \text { Ar ring }+\delta \mathrm{HCC}(11 \%) \text { Ar ring+ } \delta \\
\mathrm{HCH}_{\text {asy }}(14 \%)\end{array}$ \\
\hline 53 & & 1257 & 1206.72 & 43.11 & $v \mathrm{NC}_{\text {asy }}(16 \%)+$ Th ring $+v \mathrm{CO}(13 \%)$ Ar ring $+\tau \mathrm{HCCN}(12 \%)$ Th ring \\
\hline 52 & & 1253 & 1202.88 & 25.58 & $\tau \mathrm{HCSC}_{\text {asy }}(84 \%)$ Th ring \\
\hline 51 & & 1212 & 1163.52 & 40.44 & $\delta \mathrm{HCH}_{\mathrm{asy}}(15 \%)+\tau \mathrm{HCOC}(44 \%)$ Ar ring \\
\hline 50 & 1148 & 1194 & 1146.24 & 22.35 & $v \mathrm{CC}(10 \%)$ Ar ring $+\delta \mathrm{HCC}(38 \%)$ Ar ring $+\tau \mathrm{HCOC}_{\mathrm{asy}}(14 \%)$ Ar ring \\
\hline 49 & & 1172 & 1125.12 & 45.03 & $\delta \mathrm{HCH}(21 \%)+\tau \mathrm{HCOC}(35 \%)$ Ar ring \\
\hline 48 & & 1171 & 1124.16 & 101.55 & $\delta \mathrm{HCC}_{\text {asy }}(13 \%)$ Ar ring $+\tau \mathrm{HCOC}_{\text {asy }}(17 \%)$ Ar ring \\
\hline 47 & & 1148 & 1102.08 & 3.08 & $\delta \mathrm{HCS}_{\text {asy }}(52 \%)$ Th ring $+\tau \mathrm{HCSC}(28 \%)$ Th ring \\
\hline 46 & & 1131 & 1085.76 & 146.72 & $v \mathrm{NC}_{\text {asy }}(15 \%)$ Th ring $+v \mathrm{CC}(11 \%)$ Th ring \\
\hline 45 & & 1111 & 1066.56 & 17.22 & $\delta \mathrm{HCH}_{\text {asy }}(12 \%)+\delta \mathrm{CCN}_{\text {asy }}(13 \%)$ Th ring $+\tau \mathrm{HCCN}(36 \%)$ Th ring asy \\
\hline 44 & & 1109 & 1064.64 & 12.00 & $v \mathrm{CC}(14 \%)$ Ar ring $+\delta \mathrm{HCC}(33 \%)$ ar ring + \\
\hline 43 & 1046 & 1075 & 1032 & 63.45 & $v \mathrm{OC}(55 \%)$ Ar ring $+\delta \mathrm{HCC}(17 \%)$ Ar ring \\
\hline 41 & & 1007 & 966.72 & 0.82 & $v \mathrm{CC}_{\mathrm{asy}}(52 \%) \mathrm{Ar}$ ring $+\delta \mathrm{CCC}_{\mathrm{asy}}(39 \%)$ Ar ring \\
\hline 40 & & 973 & 934.08 & 4.63 & $v \mathrm{CC}_{\text {asy }}(33 \%)$ Th ring \\
\hline 39 & 927 & 968 & 929.28 & 0.03 & $\tau \mathrm{HCCC}(77 \%)$ Ar ring \\
\hline 38 & 896 & 940 & 902.4 & 27.82 & $v \mathrm{NC}(10 \%)$ Ar ring $+v \mathrm{OC}_{\text {asy }}(10 \%)$ Ar ring $+v \mathrm{CC}_{\text {asy }}(27 \%)$ Th ring \\
\hline 37 & & 922 & 885.12 & 7.21 & $\begin{array}{l}\delta \text { HCS }(45 \%) \text { Th ring }+\tau \operatorname{HCSC}(21 \%) \text { Th ring }+\tau C C C_{\text {asy }}(19 \%) \text { Th ring+ } \\
\gamma \operatorname{OCNC}_{\text {asy }}(15 \%)\end{array}$ \\
\hline 36 & & 891 & 855.36 & 29.40 & $v \mathrm{CC}_{\mathrm{asy}}(12 \%)$ Th ring $+\delta \mathrm{NCN}_{\mathrm{asy}}(10 \%)+\tau \mathrm{HCCC}(12 \%)$ Ar ring \\
\hline 35 & & 872 & 837.12 & 7.58 & $\tau H C C C(68 \%)$ Ar ring \\
\hline 34 & & 863 & 828.48 & 5.52 & $\tau \mathrm{HCCC}(68 \%)$ Ar ring \\
\hline 33 & 775 & 791 & 759.36 & 5.21 & $\delta \mathrm{NCN}_{\text {asy }}(32 \%)$ Th ring $+\tau \mathrm{HCNC}(33 \%)$ Th ring \\
\hline 32 & & 782 & 750.72 & 30.71 & $\begin{array}{l}\tau \mathrm{HCCC}(17 \%) \text { Ar ring }+\tau \mathrm{HCCC}_{\text {asy }}(17 \%) \text { Ar ring }+\tau \mathrm{CCCC}_{\text {asy }}(29 \%) \\
\text { Ar ring }\end{array}$ \\
\hline 31 & 740 & 779 & 747.84 & 20.41 & $\delta \mathrm{CNC}(27 \%)$ Th ring \\
\hline 30 & 704 & 751 & 720.96 & 8.75 & $v \mathrm{CC}_{\text {asy }}(10 \%)$ Ar ring $+v \mathrm{CC}(10 \%)$ Th ring $+v \mathrm{SC}(21 \%)$ Th ring \\
\hline 29 & & 705 & 676.8 & 22.45 & $\tau \mathrm{HCCC}_{\text {asy }}(56 \%)$ Ar ring $+\tau \mathrm{CCCC}_{\text {asy }}(10 \%)$ Ar ring \\
\hline 28 & 647 & 669 & 642.24 & 0.28 & $v \mathrm{SC}(10 \%)$ Th ring $+\tau \mathrm{HCCC}_{\mathrm{asy}}(12 \%)$ Ar ring $+\tau \mathrm{CCCC}(43 \%)$ ar ring \\
\hline 27 & 609 & 643 & 617.28 & 4.95 & $v \mathrm{CC}(28 \%)$ Th ring $+\delta \mathrm{CCC}_{\text {asy }}(11 \%)$ Ar ring \\
\hline
\end{tabular}




\begin{tabular}{|c|c|c|c|c|c|}
\hline 26 & & 617 & 592.32 & 4.44 & $\gamma \mathrm{SNNC}_{\text {asy }}(54 \%)$ Ar ring \\
\hline 25 & 584 & 610 & 585.6 & 5.88 & $\delta \mathrm{CNC}_{\text {asy }}(11 \%)$ Th ring \\
\hline 24 & & 593 & 569.28 & 17.41 & $\delta \mathrm{CCC}_{\text {asy }}(14 \%)$ Ar ring $+\gamma \mathrm{OCNC}(19 \%)$ Th ring \\
\hline 23 & & 581 & 557.76 & 1.53 & $\tau \mathrm{HCSC}_{\text {asy }}(16 \%)$ Th ring $+\gamma \mathrm{OCNC}_{\text {asy }}(30 \%)$ Th ring \\
\hline 22 & 526 & 544 & 522.24 & 3.20 & $v \mathrm{SC}(12 \%)$ Th ring $+\delta \mathrm{CCC}(25 \%)$ Ar ring $+\tau \mathrm{CCCC}(12 \%)$ Ar ring \\
\hline 21 & & 503 & 482.88 & 23.21 & $v \mathrm{SC}_{\mathrm{asy}}(23 \%) \mathrm{Th}$ ring $+\delta \mathrm{CCN}_{\mathrm{asy}}(14 \%)$ Ar ring \\
\hline 20 & & 484 & 464.64 & 0.63 & $\delta \mathrm{CNC}_{\text {asy }}(10 \%)$ Ar ring $+\delta \mathrm{CCO}_{\text {asy }}(32 \%)$ Ar ring \\
\hline 19 & & 471 & 452.16 & 16.37 & $\delta \mathrm{CNC}_{\text {asy }}(19 \%)$ Th ring $+\delta \mathrm{CCN}_{\text {asy }}(16 \%)$ Th ring $+\delta \mathrm{CCO}_{\text {asy }}(12 \%)$ Th ring \\
\hline 18 & & 454 & 435.84 & 1.08 & $\delta \mathrm{CCO}_{\text {asy }}(11 \%) \mathrm{Ar}$ ring $+\tau \mathrm{CCCC}_{\text {asy }}(36 \%)$ Ar ring \\
\hline 17 & & 403 & 386.88 & 2.62 & $\delta \mathrm{CCC}(12 \%)$ Ar ring $+\delta \mathrm{COC}_{\mathrm{asy}}(21 \%)$ Ar ring \\
\hline
\end{tabular}

$\nu$ : stretching(elongation); sy: symmetric; asy: asymmetric; $\beta$ : in plane bending; $\gamma$ : out-of-plane bending, $\omega$ : wagging; $\tau$ : twisting; $\delta$ : bending; $\rho$ : rocking; vibrational modes are based on potential energy distribution (PED) and only contributions over $10 \%$ are given; scaled frequencies are in unit of $\mathrm{cm}^{-1} ; \mathrm{I}_{\mathrm{IR}}$ infrared intensities are in unit of $\mathrm{km} \mathrm{mol}^{-1}$.

down by scaling the previous values to $902(10 \%), 1348$ (13\%), 1302 (46\%), 1248 (12\%), 1206 (16\%), 1085 (15\%), respectively with the percentage of the PED contribution indicated between brackets.

\section{4. ${ }^{1} \mathrm{H}$ and ${ }^{13} \mathrm{C}$ NMR Calculations}

To calculate isotropic chemical shifts $(\delta)$ with respect to tetramethylsilane (TMS): $\delta^{\mathrm{x}}{ }_{\text {iso }}=\sigma^{\mathrm{TMS}}{ }_{\text {iso }}-\sigma^{\mathrm{x}}{ }_{\text {iso, }}{ }^{34}$ isotropic shielding values 184.2796 and $32.2899 \mathrm{ppm}$ of $\sigma^{\mathrm{TMS}}$ iso were used for $\mathrm{C}$ and $\mathrm{H}$ NMR spectra, respectively. It is known that the range of ${ }^{13} \mathrm{C}$ NMR chemical shifts for analogous organic molecules usually are $>100 \mathrm{ppm}^{35,36}$ Methanol solvent has been used for chemical shift measurements. The atoms were labeled according to the numbering presented in Figure 1 (B). Calculated and experimental values for ${ }^{1} \mathrm{H}$ and ${ }^{13} \mathrm{C}$ NMR are collected in Table 7 . In this research, aromatic carbons give signals in overlapped areas of the spectrum with chemical shift values from 106 to $170 \mathrm{ppm}$ while experimental chemical shift values of aromatic carbon atoms are in the range 107 to $160 \mathrm{ppm}$ as they would be expected (Table 7). Carbon atoms (C1, C4 and C6) attached to the $\mathrm{N}$ atom have larger chemical shifts due to the more electronegative property of the $\mathrm{N}$ atom which polarizes the electron distribution in its binding to the atom adjacent carbon and reduces the value of the chemical shifts. Usually, the chemical shifts obtained and calculated for the ${ }^{1} \mathrm{H}$ atoms of the methyl groups are quite weak. The maximum chemical shift value for all the hydrogen atoms is $7.24 \mathrm{ppm}$.

\section{5. Frontier Molecular Orbitals (FMOs)}

The highest occupied molecular orbitals (HOMO) and the lowest unoccupied molecular orbitals (LUMO) are named as frontier molecular orbitals (FMO). The HOMO represents the ability to donate an electron, whereas LUMO as an electron acceptor represents the ability to obtain an electron. ${ }^{37}$ The calculation of these parameters is very important because it allowed us to verify a lot of properties, such as the kinetic stability and chem-
Table 7. Experimental and calculated ${ }^{1} \mathrm{H}$ and ${ }^{13} \mathrm{C}$ NMR data for $(Z)$ 3- $N$-(ethyl)-2- $N$ '-((3-methoxyphenyl)imino)thiazolidine-4-one.

\begin{tabular}{lcc}
\hline Chemical shifts & & \\
\hline${ }^{1} \mathrm{H}$ & Experimental & B3LYP/6-311G(d) \\
$\mathrm{H}_{51}$ & 3.78 & 4.03 \\
$\mathrm{H}_{52}$ & 3.78 & 3.94 \\
$\mathrm{H}_{21}$ & 3.90 & 4.49 \\
$\mathrm{H}_{22}$ & 3.90 & 4.25 \\
$\mathrm{H}_{31}$ & 1.29 & 1.02 \\
$\mathrm{H}_{32}$ & 1.29 & 1.92 \\
$\mathrm{H}_{33}$ & 1.29 & 1.19 \\
$\mathrm{H}_{9}$ & 6.52 & 6.26 \\
$\mathrm{H}_{7}$ & 6.67 & 6.71 \\
$\mathrm{H}_{11}$ & 6.56 & 6.64 \\
$\mathrm{H}_{10}$ & 7.24 & 7.42 \\
$\mathrm{H}_{121}$ & 3.79 & 4.32 \\
$\mathrm{H}_{122}$ & 3.79 & 3.74 \\
$\mathrm{H}_{123}$ & 3.79 & 3.73 \\
${ }^{13} \mathrm{C}$ & & \\
$\mathrm{C} 1$ & 154.23 & 158.27 \\
$\mathrm{C} 2$ & 32.76 & 37.78 \\
$\mathrm{C} 3$ & 12.52 & 15.06 \\
$\mathrm{C} 4$ & 171.55 & 176.61 \\
$\mathrm{C} 5$ & 38.26 & 37.57 \\
$\mathrm{C} 6$ & 149.32 & 158.15 \\
$\mathrm{C} 7$ & 110.39 & 113.89 \\
$\mathrm{C} 8$ & 160.37 & 170.89 \\
$\mathrm{C} 9$ & 107.22 & 106.29 \\
$\mathrm{C} 10$ & 129.99 & 136.22 \\
$\mathrm{C} 11$ & 113.20 & 120.10 \\
$\mathrm{C} 12$ & 55.25 & 58.70 \\
\hline & &
\end{tabular}

ical reactivity. Figure 5 shows the atomic orbital HOMOLUMO plot of the frontier molecular orbitals computed at B3LYP/6-311G(d,p) level for the title compound. As shown in Figure 5, in HOMO density, electrons are mainly located on the methoxyphenyl group. However, when an electron transition occurs, the electron enters into the LUMO, and then the electron will mainly be localized on thiazole ring and carboxylic group. The positive phase is red and the negative one is green coloured. The HOMO- 


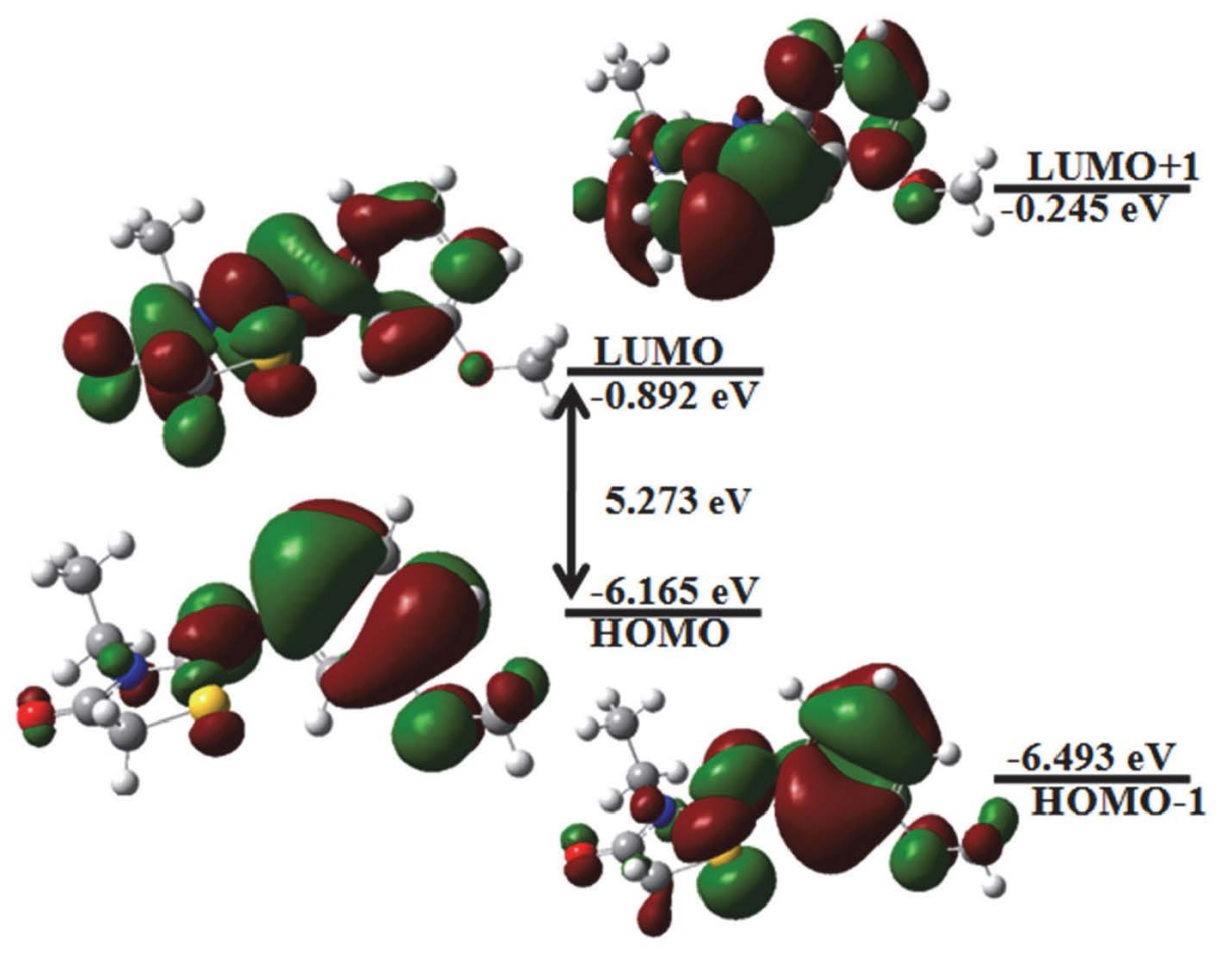

Figure 5. Electron distribution of the HOMO-1, HOMO, LUMO and LUMO+1 energy levels for the title molecule.

LUMO energy gap of our molecule was calculated at the B3LYP/6-311G (d,p) level with HOMO energy $-6.165 \mathrm{eV}$, LUMO energy $-0.892 \mathrm{eV}$ and HOMO-LUMO energy gap $5.273 \mathrm{eV}$. The second highest occupied MO (HOMO-1) and the second lowest unoccupied $\mathrm{MO}(\mathrm{LUMO}+1)$ were calculated using the same level of theory. 3D plots of the HOMO-1, HOMO, LUMO and LUMO+1 orbitals of the studied molecule are drawn in Figure 4.

\section{6. Chemical Reactivity}

Global chemical reactivity descriptors (GCRD) parameters are a good indication to highlight the relationship between chemical reactivity and strength of structure. As are mentioned in literature, GCRD parameters can be obtained by using the following equations: $\eta=\frac{1}{2}\left(E_{\text {LUм }}-E_{\text {номо }}\right)$; $\mu=-\left(\frac{I+A}{2}\right) ; S=\frac{1}{2 \eta} ; \quad \chi=\left(\frac{I+A}{2}\right) ; \omega=\frac{\mu^{2}}{2 \eta} . A=-E_{L U M O}$ and $I=-E_{\text {номо }}$. The electron affinity $(A)$ and ionization potential $(I)$ can be evaluated as and. The calculated values of GCRD parameters for the title molecule are summarized in Table 8.

The chemical hardness $(\eta)$ value is $2.636 \mathrm{eV}$ indicating that the charge transfer occurs within the molecule. From Table 8, the electrophilic behaviour of the molecule is confirmed by the global electrophilicity index $(\omega)$ which has a greater value of $2.361 \mathrm{eV}$. On the other hand, the chemical stability of the title molecule is explained by the chemical potential $(\mu)$ value which is $-3.528 \mathrm{eV}$.
Table 8. Calculated energy values of the title compound by B3LY$\mathrm{P} / 6-311 \mathrm{G}(\mathrm{d}, \mathrm{p})$ method.

\begin{tabular}{lc}
\hline Parameters & Calculated energies \\
\hline$E_{\text {HOMO }}$ & -6.165 \\
$E_{\text {LUMO }}$ & -0.892 \\
Energy gap $(\Delta E)$ & 5.273 \\
Ionization potential $(I)$ & 6.165 \\
Electron affinity $(A)$ & 0.892 \\
Electronegativity $(\chi)$ & 3.528 \\
Chemical potential $(\mu)$ & -3.528 \\
Chemical hardness $(\eta)$ & 2.636 \\
Chemical softness $(s)$ & 0.189 \\
Electrophilicity index $(\omega)$ & 2.361 \\
\hline
\end{tabular}

\section{7. Electrostatic Potential}

In a crystal, the location of positive and negative charges allowed us to define very interesting physical properties such as the molecular electrostatic potential (MEP). ${ }^{38}$ Nowadays, theoretical and experimental MEP surfaces are determined using quantum chemistry and $\mathrm{X}$-ray diffraction. ${ }^{39-42}$ The MEP around the title molecule was calculated from the total density (TD) for the title compound, the two maps (TD and MEP) are represented in the Figure 6. From the figure it can be seen that the negative MEP is related to the electronegativity and partial charges of oxygen atoms $\mathrm{O} 1$ and $\mathrm{O} 2$. The blue color in Figure $6 \mathrm{~B}$ gives the maximum positive region as the preferred 

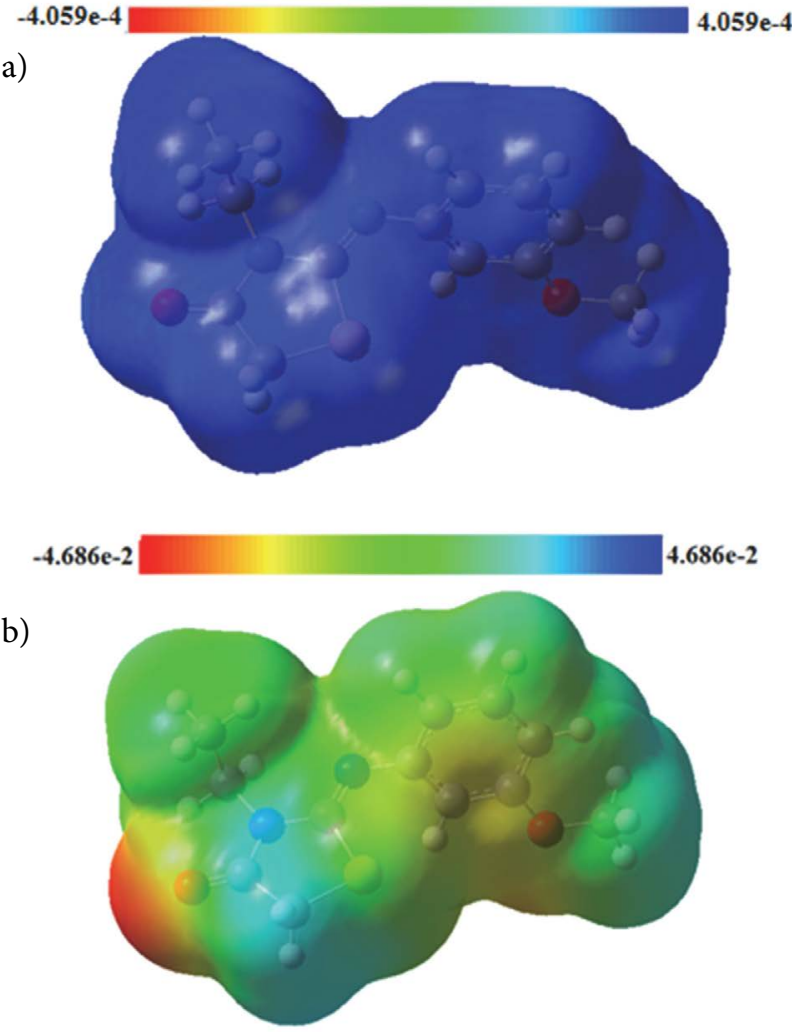

Figure 6. MEP plots for (Z)-3-N-(ethyl)-2-N'-((3-methoxyphenyl) imino)thiazolidine-4-one: (a) total electron density, (b) MEP surface.

site for nucleophilic attack. In terms of color, the MEP plot lies in the fact that it simultaneously displays molecular size, shape as well as positive, negative and neutral electrostatic potential regions (Figure 6). The MEP indicate well the relationship between the molecular structure and these physico-chemical properties. ${ }^{43-47}$ Among them, the molecular dipole moment can be calculated. The orientation of the molecular dipole moment for the title compound is represented in Figure 7. This orientation confirms the elec-

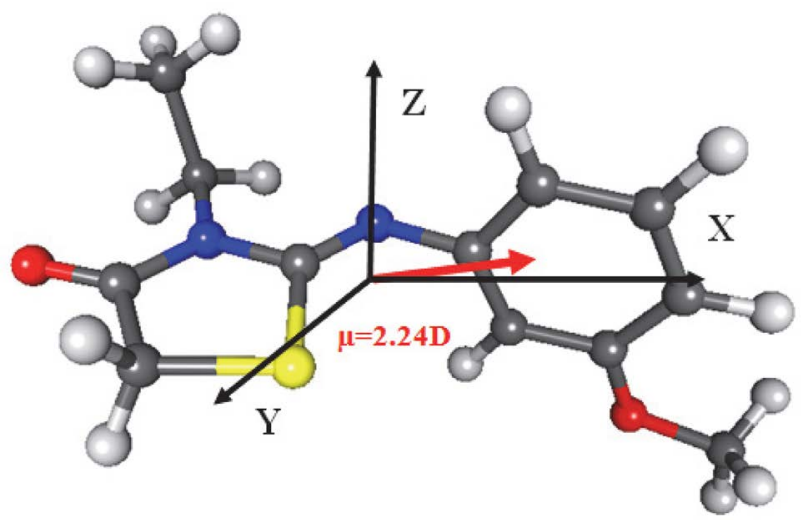

Figure 7. Orientation of the molecular dipole moment from DFT calculation. trostatic distribution previously defined. The calculated dipole moment value is $2.24 \mathrm{D}$.

\section{Conclusion}

In this study, (Z)-3-N-(ethyl)-2- $N$ '-((3-methoxyphenyl)imino)thiazolidine-4-one was synthesized and characterized by IR, ${ }^{1} \mathrm{H}$ and ${ }^{13} \mathrm{C}$ NMR and X-ray single-crystal diffraction techniques. The crystal structure determination shows that the title compound crystallizes in monoclinic system with space group C2/c. Theoretical calculations of the molecular structures, wavenumbers and NMR spectra of the title compound have been carried out using DFT-B3LYP/6-311G $(\mathrm{d}, \mathrm{p})$. As result, the experimental and the optimized crystal structures of the title compound were slightly different. Most of the experimental bond lengths and bond angles are slightly larger than the optimized values. These differences are due to that the theoretical calculations are performed for isolated molecule in gaseous phase and the experimental results are for a molecule in the crystalline environment. The geometry of the solid-state structure is subject to intermolecular forces, such as Van der Waals interactions and crystal packing contacts. Comparison between the chemical shifts and the experimental data shows a very good agreement for both ${ }^{1} \mathrm{H}$ and ${ }^{13} \mathrm{C}$ NMR shift values. The HOMO-LUMO gap and chemical reactivity descriptors indicate that the compound is more stable. The general conclusion from the estimation of the dipolar moment $(2.24 \mathrm{D})$ and the electrostatic potential of ((Z)-3-N-(ethyl)-2-N'-((3-methoxyphenyl)imino)thiazolidine-4-one molecule is that the region of the thiazolidinone group is electronegative and the methyl and hydrogen atoms connected to the benzene ring are electropositive.

\section{Supplementary Material}

Crystallographic data for the structure reported in this article have been deposited with Cambridge Crystallographic Data Center, CCDC 1871013. Copies of this information may be obtained free of charge from the Director, CCDC, 12 Union Road, Cambridge, CB2 1EZ, UK. Facsimile (44) 01223336 033, E-mail: deposit@ccdc.cam. ac.uk or http//www.ccdc.com.ac.uk/deposit.

\section{References}

1. J. Quiroga, P. Hernandez, B. Insuasty, R. Abonia, J. Cobo, A. Sanchez, M. Nogueras, J. Chem. Soc. Perkin Trans. 1 2002, 4, 555-559. DOI:10.1039/b109676a

2. I. Hutchinson, S. A. Jennings, B. R. Vishnuvajjala, A. D. Westwell, M. F. G. Stevens, J. Med. Chem. 2002, 45, 744-747.

DOI:10.1021/jm011025r 
3. R. I. Bahoussi, A. Djafri, A. Chouaih, A. Djafri, F. Hamzaoui, Acta Cryst. 2017, E73, 173-176.

DOI:10.1107/S205698901700041X

4. A. Srinivas, M. Sunitha, P. Karthik, K. V. Reddy, Acta Chim. Slov. 2017, 64, 1030-1041. DOI:10.17344/acsi.2017.3805

5. K. D. Hargrave, F. K. Hess, J. T. Oliver, J. Med. Chem. 1983, 26, 1158-1163. DOI:10.1021/jm00362a014

6. W. C. Patt, H. W. Hamilton, M. D. Taylor, M. J. Ryan, J. R. Taylor, C. J. C. Connolly, J. Med. Chem. 1992, 35, 2562-2572. DOI:10.1021/jm00092a006

7. R. N. Sharma, F. P. Xavier, K. K. Vasu, S. C. Chaturvedi, S. S. Pancholi, J. Enz. Inhib. Med. Chem. 2009, 24, 890-897. DOI:10.1080/14756360802519558

8. J. C. Jaen, L. D. Wise, B. W. Caprathe, H. Tecle, S. Bergmeier, C. C. Humblet, T. G. Heffner, J. Med. Chem. 1990, 33, 311317. DOI:10.1021/ jm00163a051

9. K. Tsuji, H. Ishikawa, J. Med. Chem. Lett. 1994, 4, 1601-1606. DOI:10.1016/S0960-894X(01)80574-6

10. F. W. Bell, A. S. Cantrell, M. Hogberg, S. R. Jaskunas, N. G. Johansson, C. L. Jordon, J. Med. Chem. 1995, 38, 4929-4936. DOI:10.1021/jm00025a010

11. N. Ergenc, G. Capan, N. S. Gunay, S. Ozkirimli, M. Gungor, S. Ozbey, E. Kendi, Arch. Pharm. 1999, 332, 343-347.

DOI:10.1002/（SICI)1521-4184(199910)332:10<343::AID -ARDP343>3.0.CO;2-0

12. J. S. Carter, S. Kramer, J. J. Talley, T. Penning, P. Collins, M. J. Graneto, K. Eibert, Bioorg. Med. Chem. Lett. 1999, 9, 11711174. DOI:10.1007/s00706-010-0392-3

13. A. Badorc, M. F. Bordes, P. De Cointet, P. Savi, A. Bernat, A. Lale, M. Petitou, J. Med. Chem. 1997, 40, 3393-3401. DOI:10.1021/jm970240y

14. G. Aridoss, S. Amirthaganesan, M. S. Kim, J. T. Kim, Y. T. Jeong, Eur. J. Med. Chem. 2009, 44, 4199-4210.

DOI:10.1016/j.ejmech.2009.05.015

15. Bruker Analytical X-ray Systems, Inc, Apex2, Version 2 User Manual, M86-E01078, 2006, 6, Madison, WI.

16. Siemens Industrial Automation, Inc. SADABS: Area-Detector Absorption Correction, 1996, Madison, WI.

17. P. W. Betteridge, J. R. Carruthers, R. I. Cooper, K. Prout, D. J. Watkin, J. Appl. Crystallogr. 2003, 36,1487.

DOI:10.1107/S0021889803021800

18. L. Palatinus, G. Chapuis, J. Appl. Crystallogr. 2007, 40, 782785. DOI:10.1107/S002188980702420X

19. C. Jelsch, B. Guillot, A. Lagoutte, C. Lecomte, J. Appl. Crystal$\log r$. 2005, 38, 38-54.

DOI:10.1107/S0021889804025518

20. A. D. Becke, J. Chem. Phys. 1997, 107, 8554-8560. DOI:10.1063/1.475007

21. P. C. R. Kumar, V. Ravindrachary, K. Janardhana, B. Poojary, J. Cryst. Growth 2012, 354, 182-187.

DOI:10.1016/j.jcrysgro.2012.06.006

22. A. Frish, A. B. Nielsen, A. J. Holder, Gaussview Users Manual, Gaussian Inc, Pittsburg, 2000.

23. M. H. Jamróz, J. C. Z. Dobrowolski, J. Mol. Struct. 2001, 565, 475-480. DOI:10.1016/S0022-2860(00)00908-X

24. L. J. Farrugia, "ORTEP-3 for windows-a version of ORTEPI-
II with a graphical user interface (GUI), J. Appl. Crystallogr. 1997, 30, 565-568. DOI:10.1107/S0021889897003117

25. S. Yahiaoui, A. Moliterni, N. Corriero, C. Cuocci, K. Toubal, A. Chouaih, A. Djafri, F. Hamzaoui, J. Mol. Struct. 2019, 1177, 186-192. DOI:10.1016/j.molstruc.2018.09.052

26. N. Khelloul, K. Toubal, N. Benhalima, R. Rahmani, A. Chouaih, A. Djafri, F. Hamzaoui, Acta. Chim. Slov. 2016, 63, 619-626. DOI:10.17344/acsi.2016.2362

27. S. P. V. Chamundeeswari, E. R. J. J. Samuel, N. Sundaraganesan, Eur. J. Chem. 2011, 2, 136-145.

DOI:10.5155/eurjchem.2.2.136-145.169

28. A. Eşme, S. Güneşdoğdu Sağdınç, Spectrochim. Acta A Mol. Biomol. Spectrosc. 2018, 188, 443-455.

DOI:10.1016/j.saa.2017.07.034

29. G. Muhammad, A. Muhammad, A. M. Khalid, J. Mol. Struct. 2018, 1160, 129-141.

DOI:10.1016/j.molstruc.2018.01.100

30. P. K. Murthy, G. Krishnaswamy, S. Armaković, S. J. Armaković, P. A.Suchetan, N. R. Desai, V. Suneetha, R. Sreenivasa Rao, G. Bhargavi, D. B. Arunakumar, J. Mol. Struct. 2018, 1162, 81-95. DOI:10.1016/j.molstruc.2018.02.081

31. A. Teimouri, A. N. Chermahini, M. D. Emami, Arkivoc 2008, $8,172-187$.

32. H. Dammak, A. Yangui, S. Triki, Y. A. H. Feki, J. Lumin. 2015, 161, 214-220. DOI:10.1016/j.jlumin.2015.01.010

33. M. Silverstein, G. C. Basseleer, C. Moril, Spectrometric Identification of Organic Compounds, Wiley, New York, 1981.

34. S. Muthu, J. U. Maheswari, T. Sundius, Spectrochim. Acta A Mol. Biomol. Spectrosc. 2013, 108, 307-318.

DOI:10.1016/j.saa.2013.02.022

35. G. Socrates, Infrared Characteristic Group Frequencies, Wiley Inter Science Publication, 1980.

36. G. Varsanyi, Vibrational Spectra of Benzene Derivates, Academic Press, New York, 1969.

37. K. Toubal, N. Boukabcha, Ö. Tamer, N. Benhalima, S. Altürk, D. Avc1, A. Chouaih, Y. Atalay, A. Djafri, F. Hamzaoui, J. Mol. Struct. 2017, 1147, 569-581.

DOI:10.1016/j.molstruc.2017.06.102

38. R. Rahmani, N. Boukabcha, A. Chouaih, F. Hamzaoui, S. Goumri, J. Mol. Struct. 2018,1155, 484-495.

DOI:10.1016/j.molstruc.2017.11.033

39. H. Benaissi, M. Drissi, S. Yahiaoui, Y. Megrouss, A. Chouaih, F. Hamzaoui, J. Optoelectron. Biomed. M. 2018, 10, 73-82

40. N. Boukabcha, A. Feddag, R. Rahmani, A. Chouaih, F. Hamzaoui, J. Optoelectron. Adv. M. 2018, 20, 140-148

41. N. Boubegra, Y. Megrouss, N. Boukabcha, A. Chouaih, F. Hamzaoui, Rasayan. J. Chem. 2016, 9, 751-761.

42. M. Drissi, N. Benhalima, Y. Megrouss, R. Rahmani, A. Chouaih, F. Hamzaoui, Molecules 2015, 20, 4042-4045. DOI: $10.3390 /$ molecules

43. J. S. Murray, K. Sen, Molecular Electrostatic Potentials, Concepts and 399 Applications, Elsevier, Amsterdam, 1996.

44. E. Scrocco, J. Tomasi, in: P. Lowdin (Ed) Advances in Quantum Chemistry, Academic Press, New York. 1978. 402.

45. F. J. Luque, M. Orozco, P. K. Bhadane, S. R. Gadre, J. Phys. 
Chem. 1993, 97, 9380-9384. DOI:10.1021/j100139a021

46. J. Sponer, P. Hobza, Int. J. Quant. Chem. 1996, 57, 959-970.

DOI:1 0.1002/(SICI) 1097-461X(1996)57:5<959::AID-

QUA16>3.0.CO;2-S
47. M. Govindarajan, M. Karabacak, Spectrochim. Acta A Mol. Biomol. Spectrosc. 2012, 96, 421-435.

DOI:10.1016/j.saa.2012.05.067

\section{Povzetek}

V prispevku poročamo o sintezi in karakterizaciji (Z)-3-N-(etil)-2- $N^{\prime}-((3-$ metoksifenil)imino)tiazolidin-4-ona z FT-IR, ${ }^{1} \mathrm{H}$ in ${ }^{13} \mathrm{C}$ NMR ter $\mathrm{z}$ rentgensko difrakcijo monokristala. Eksperimentalna potrditev kristalne strukture temelji na pridobljenih rentgenskih difrakcijskih podatkih. Pomembna značilnost strukture je obstoj dihedralnega kota, ki ga tvorita ravnini benzenskega in tiazolidinonskega obroča, $v$ vrednosti $86.0^{\circ}$, kar kaže na odsotnost $\pi-\pi$ interakcij, hkrati pa nakazuje na neplanarno strukturo. V kristalu so molekule povezane s $\mathrm{C}-\mathrm{H} \cdots \mathrm{O}$ in $\mathrm{C}-\mathrm{H} \cdots \mathrm{N}$ vodikovimi vezmi, ki so odgovorne za trodimenzionalno molekulsko pakiranje v strukturi. Da bi lahko eksperimentalne rezultate primerjali s teoretično izračunanimi, smo izvedli kvantno kemijske DFT izračune s pomočjo B3LYP/6-311G(d,p) baznega seta. Ob tem smo izračunali še elektrostatski potencial okoli molekule ter HOMO in LUMO energijske nivoje. Ugotovili smo orientacije dipolnih momentov in s tem razkrili naravno inter- in intramolekularnih prenosov naboja. Nazadnje smo s pomočjo izračuna deskriptorjev kemijske reaktivnosti potrdili še stabilnost opisane spojine. 